Dept. of Surgery, Anesthesiology and Radiology,

Fac. Vet. Med., Beni-Suef Univ.

\title{
RETROGRADE CATHETERIZATION OF THE URINARY BLADDER OF CALVES
}

(With 2 Tables and 9 Figures)

By

\author{
A.M. SEDEEK; H.A. BAKR* and M.S. HASSAN*
}

* Dept of Internal medicine, Fac. Vet. Med., Beni-Suef Univ.

(Received at 28/2/2009)

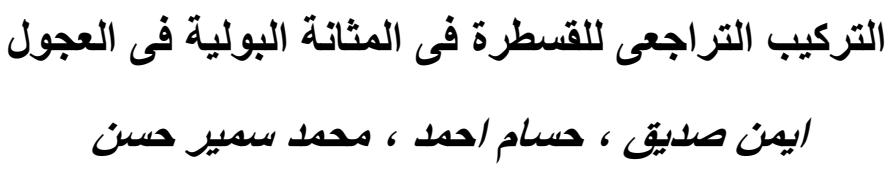

تم فى هذه الدر اسة توصيف عملية التركيب التر اجعى للقسطرة فى المثانة البولية فى العجول

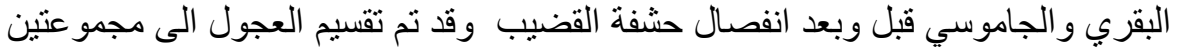

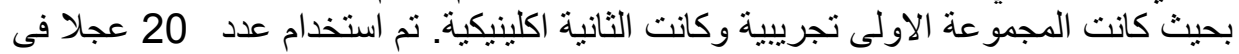

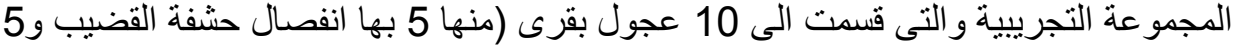

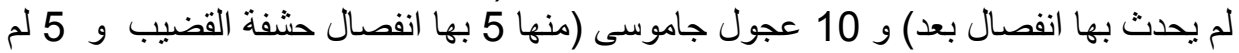

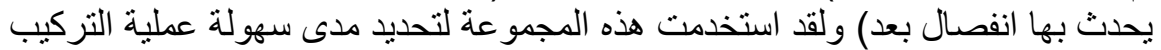

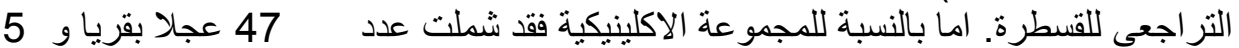

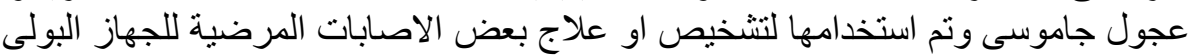

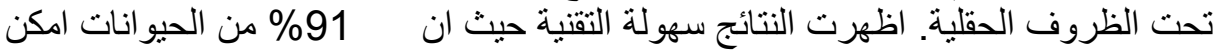

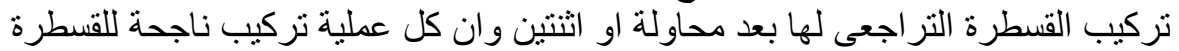

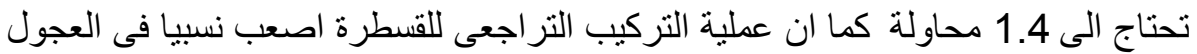

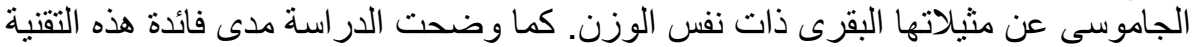

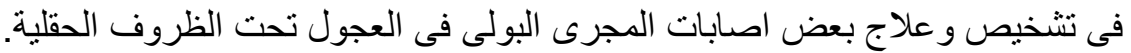

\section{SUMMARY}

A simple technique for catheterization of the urinary bladder of cattle and buffalo calves before and after separation of glans penis was described. The study was classified into two main parts. Part I was experimentally conducted on 10 cattle and 10 buffalo calves and each group was subdivided into 5 animals with and 5 animals without separation of glans penis, and it was used for determination of the ease of retrograde catheterization of the urinary bladder. Part II was clinically conducted on 47 cattle and 5 buffalo calves either for diagnosis or 
treatment of certain affections. Results revealed the easy catheterization of the urinary bladder in $91 \%$ of cases after 1-2 trials, and that one successful retrograde catheterization requires 1.4 trials. It was observed that the catheterization was more difficult in buffalo calves than cattle ones. The technique was useful for diagnosis and treatment of certain affections of urinary tract in cattle and buffalo calves.

Key wards: Catheterization, urinary bladder, cattle, buffalo, calves

\section{INTRODUCTION}

Despite the frequent need for retrograde catheterization of the urinary bladder in male ruminants, it is too difficult or even impossible, and too dangerous procedure because of the risk of injury at the bulbourethral region (Rosenberger, et al., 1979), and this in fact is due to certain anatomical considerations, one of these anatomical considerations is the urethral recess or diverticulum, which is a small pouch of the urethra at ischiatic arch observed in animal species with fibroelastic penis. Presence of is diverticulum and its dorsal location to the urethra predispose the catheter to be directed into the diverticulum, as it passes dorsally around the ischiatic arch, and accordingly makes retrograde catheterization of the urinary bladder very difficult or often impossible (Hinkle, et al., 1978; Bezuidenhout and Coetzer, 1982; Garrett, 1987; Timm and Watrous, 1988; Gasthuys, et al., 1993 and Radostits, et al., 2000). Sigmoid flexure also acts as an obstacle for catheterization of that species and trials were performed to over come that problem either by retractor penis myotomy in sheep and goat (Shokry and Al-Saadi, 1980), or by adoption of pudendal nerve block in adjunction with ataractic drugs, in bulls, to induce relaxation of retractor penis muscle (Kelly, 1984). However the difficulty of retrograde catheterization increases in younger animals than elder ones and it is very difficult to achieve that procedure in prepubertal animals as the frenulum limits exposure of the glans penis, and exteriorization of the penis becomes very difficult Van Metre (1987).

Bulls can't usually be stimulated to pass urine by bladder massage, and a more effective method is to wash the outside of the prepuce with warm water, but such urine sample is nearly always contaminated and unsuitable for bacteriological, chemical, and microscopical testing (Rosenberger, et al., 1979 and Kelly, 1984). However, retrograde catheterization permits aseptic collection of urine sample in calves and bulls for microbiological examination (Radostits, et 
$a l ., 2007)$ or for analysis of minerals or trace elements in urine (Ibrahim, 2002). Aside from this, there are various uses of that technique including; application of contrast studies of bladder and urethra in animals suspected of urolithiasis; differentiation between cystitis and obstructive urolithiasis; reduction of possibility of urethrostomy either via flushing of obstructed urethra or by using urethral endoscopy that expanded the therapeutic options for management of urolithiasis by providing a route for conducting laser lithotripsy; pushing of the stone back to the bladder; and detection of seat of obstructing urethrolith (Bryden, 1984; Van Weeren, et al., 1987; Van Metre and Smith 1991; Halland, et al., 2002; Seif, et al., 2007; and Van Metre).

Unfortunately, the exact technique for performing such procedure and how to over come the difficulties had little attention and there is no available literature discussing it. However the long flexible catheter could be improved to gain some rigidity by using stilette so that it could be easily advanced through the resisting urethra (Lavania and Angelo, 1975; Abd Elfattah and Sedeek, 2005; and Seif, et al., 2007) or by lead wire (Blood and Henderson, 1976) that wasn't flexible enough. The urethral diverticulum could be avoided, in one study in goat, by the use of a pre-curved catheter (Van Weeren, et al., 1987); and the Sshaped sigmoid flexure could be relaxed by pudendal nerve block in adjunction with ataractic drugs (Hall and Clarke, 1983 and Kelly, 1984).

The aims of this study are to establish a simple technique for catheterization of cattle and buffalo calves urinary bladder before and after separation of glans penis, and to evaluate it under field condition in our localities.

\section{MATERIALS and METHODS}

\section{Part I (Experimental retrograde catheterization of the bladder): -}

Ten cattle calves and ten buffalo calves (each group was subdivided into 5 animals with and 5 animals without separation of glans penis) were used in this experimental part. These animals were from the farm of faculty of veterinary medicine, Beni-Suef University (Table: 1). These animals were subjected to epidural analgesia by lidocaine $2 \%$ and tranquilization by intravenous diazepam $0.5 \%$ according to Hall and Clarke (1983), then the animals were kept in lateral recumbency on the right side and the left hind limb was grasped forward and upward toward the rumen. A Rayel' s tube, of suitable size (No. 8, 10, or 12) according to animal weight, was supported with flexible wire stilette after its 
lubrication with oily antibiotic, and a toothed forceps fixed the catheter to the wire stilette $2 \mathrm{~cm}$ anterior to the hub of the catheter to avoid slippage of the wire stilette from the catheter during catheterization and the subsequent injury to the urethra. The catheter with the stilette was curved, 2-3 cm from its free end, in an angle of 90 degree to avoid catching of the tip of the catheter at the urethral recess and to facilitate its insertion into the bladder. The penis was exteriorized manually and the external urethral opening was detected (in animals had separation of glans penis) then the previously prepared tube with its stilette was lubricated and inserted into the urethra and advanced gently till reaching ischiatc arch that indicated by sudden flicking of the animal. Then the catheter was gently advanced into the bladder but when resistance was felt, the catheter was retracted backward then the forceps was rotated along the catheter for 3-5 rounds and the tube was re-advanced gently to enter the bladder (Fig. 1-4). This procedure was repeated 10 times for each animal and the frequency of entrance of the tube to the bladder was recorded for each catheterization and for each animal to make 200 successful catheterizations. Sonographic imaging was performed while the catheter was inside the bladder (Fig. 5-8) by using a portable ultrasound (240 PARUS VET Pie Medical equipment B. V. Philipsweg 1 6227Maastricht, Netherlands). Two types of transducers were used; curved array electronic transducer $(3.5-5 \mathrm{MHz})$ for examination of the bladder from exterior; and linear array electronic transducer $(6-8 \mathrm{MHz})$ for rectal examination of the bladder. These animals were monitored, for 4 weeks post catheterization, for any signs of affection of urinary tract.

\section{Part II (Clinical uses of retrograde catheterization): -}

\section{1-Diagnostic use in calves with urine retention: -}

Forty cattle calves and two buffalo calves suffered from urine retention and ruptured bladder, were subjected to catheterization in the same manner to locate the site of obstruction prior to surgical intervention (Table: 2).

History of these animals included anurea for 3-4 days and clinical signs included depression, dehydration, absence of urethral pulsation, abdominal distention with positive tactile percussion, and abdominocentesis revealed presence of uroperitonieum.

\section{2-Therapeutic use in calves with urine retention: -}

The technique was used for removal of friable stones from the urethra in 3 buffalo calves (Table: 2 and Figure 9). 


\section{3-Diagnostic use in calves with suspected pyloric obstruction: -}

Three cattle calves suffered from absence of both urination and defecation and abdominal distension and suspected to have pyloric obstruction (Table: 2), these animals were subjected to retrograde catheterization for detection of patency of urethra and bladder.

\section{4-Therapeutic medical use in calves with cystitis: -}

Four cattle calves had signs of cystitis (Table: 2); two of which had history of urine retention and ruptured bladder and they were subjected to laparotomy, suturing of the bladder, and prolonged catheterization. These animals were subjected to collection of urine sample for performing sensitivity test. Signs of cystitis included fever, frequent painful urination, and the voided small amount of urine left pus flakes at the preputial hair.

\section{RESULTS}

\section{Part I (Experimental retrograde catheterization of the bladder): -}

Results revealed that 1.4 trials were needed for every successful retrograde catheterization of the bladder (287 trials / 200 successful catheterizations) (Table 1). In $76.5 \%$ of the trials the catheter passed the pelvic flexure with one trial, and in $14.5 \%, 3 \%, 2.5 \%, 2 \%$, and $1.5 \%$ of trials, the catheter passed after 2, 3, 4, 5, and 6 trials respectively.

One successful retrograde catheterization required 1.38 trials in cattle calves, and 1.49 trials in buffalo calves, and in cattle and buffalo calves without separation of glans penis it required 1.42 and 1.52 respectively, while in cattle and buffalo calves with separation of glans penis it required 1.34 and 1.46 trials respectively.

Monitoring of animals for 4 weeks revealed no affection of the urinary tract.

\section{Part II (Clinical use of retrograde catheterization): -}

\section{1-Diagnostic use in calves with urine retention: -}

With respect to this group, the seat of obstruction could be detected in $100 \%$ of cases, and it was found that 32 animals (76.19\%) had obstructing stones at the sigmoid flexure, 9 animals $(21.43 \%)$ had stones at perineum, and one animal $(2.38 \%)$ had stones at pelvic urethra.

\section{2-Therapeutic use in calves with urine retention: -}

Three buffalo calves with signs of urine retention and intact bladder were subjected to retrograde catheterization, and during catheterization these animals voided red urine and white friable sandy materials (Fig. 9). 


\section{3-Diagnostic use in calves with suspected pyloric obstruction: -}

Three cattle calves suffered from absence of urination and defecation, abdominal distension, and sunken eyes were subjected to retrograde catheterization for confirmatory diagnosis of suspected pyloric obstruction, however, catheterization revealed patency of urethra and bladder.

\section{4-Therapeutic medical use: -}

Sensitivity test of urine sample from the bladder of these 4 cattle calves was helpful for rapid recovery and relieve of inflammatory signs of cystitis in these animals.

\section{LEGENDS OF FIGURES}

Fig. 1: Catheter in situ in cattle calf before glans separation.

Fig. 2: Catheter in situ in cattle calf after glans separation. S: Stillet, C: Catheter

Fig. 3: Catheter in situ in buffalo calf before glans separation.

Fig. 4: Catheter in situ in buffalo calf after glans separation.

Fig. 5: Ultrasonographic image of catheter in situ in cattle calf before glans separation. B: bladder, C: catheter

Fig. 6: Ultrasonographic image of catheter in situ in cattle calf after glans separation. B: bladder, C: catheter

Fig. 7: Ultrasonographic image of catheter in situ in buffalo calf before glans separation. B: bladder, C: catheter

Fig. 8: Ultrasonographic image of catheter in situ in buffalo calf after glans separation. B: bladder, C: catheter

Fig. 9: Catheter in situ in buffalo calf after glans separation. Stone was removed and bloody urine evacuated 
Assiut Vet. Med. J. Vol. 55 No. 121 April 2009 
Assiut Vet. Med. J. Vol. 55 No. 121 April 2009

Table 1: Animals used for experimental retrograde catheterization

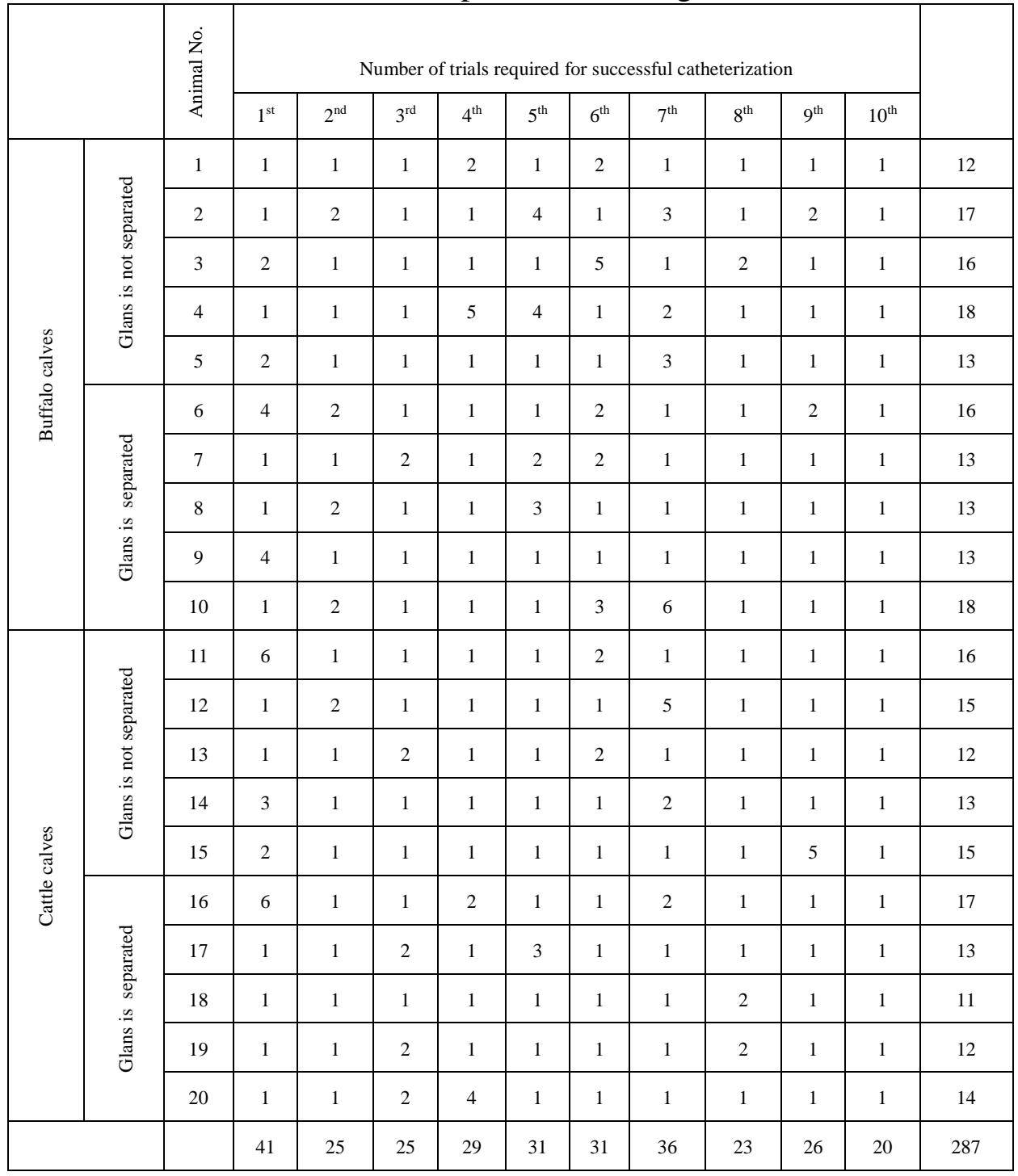

Table 2: Clinical cases used

\begin{tabular}{|c|c|c|}
\hline & Cattle calves & Buffalo calves \\
\hline Diagnostic use in calves with urine retention & 40 & 2 \\
\hline Therapeutic use in calves with urine retention & & 3 \\
\hline Diagnostic use in cases of pyloric obstruction & 3 & \\
\hline Therapeutic medical use in calves with cystitis & 4 & \\
\hline \multirow{2}{*}{ Total } & 47 & 5 \\
\hline & \multicolumn{2}{|c|}{52} \\
\hline
\end{tabular}




\section{DISCUSSION}

Regarding experimental group, the number of needed trials for every successful catheterization was 1.4 and this low number of trials might be due to the pre-curving of the catheter and stilette (Van Weeren, et al., 1987) but this is not in agreement with Blood and Henderson (1976) and this might be due to the lead nature of the stilette used as it was not flexible enough to make the passage of the catheter easy. However, retrograde catheterization was more difficult in animals that had no separation of glans penis than those with separated one Van Metre (1987). It was observed that catheterization was more difficult in buffalo calves than cattle ones, and it was observed that buffalo needed smaller size catheter than cattle calves of the same weight and this might be due to the narrower urethra and the smaller penis size in buffalo than cattle.

During catheterization, the catheter passed easy till reaching the ischiatic arch, by then animal showed flicking then the catheter either passed to the bladder or entrapped in the diverticulum and this might be due to the ventral location of the urethra with respect to the diverticulum that caused the catheter to be directed into the diverticulum and this may cause unnecessary trauma of the urethra (Hinkle, et al., 1978), however such complication could be avoided by traction of the catheter few centimeters backward, rotation of the forceps (catching the catheter) 3-5 rounds, and re-advancing the catheter.

Generally, stones located from glans penis to sigmoid flexure can be detected by palpation (Radostits, et al., 2000), but catheterization is of value need when these stones are higher. Under field conditions, the technique determined the location of the uretholithes prior to surgery in $100 \%$ of admitted cases, even it was able to detect urethrolith in the pelvic urethra in one case and accordingly the decision to make permanent tube cystotomy was taken prior to laparotomy, and during laparotomy, normograde catheterization failed and this proved the preliminary diagnosis of obstruction of pelvic urethra (Nancy, 1995; Seif, et al., 2005; and Seif, et al., 2007).

The nature of the stone in buffalo calves differs from those of cattle calves in our locality. Those of buffalo are white in color and friable and catheterization was very helpful in this species as it was able to remove stones from urethra and surgery could be avoided. However the observed friable nature of stones in buffalo is in agreement with 
Hungerford (1990) who stated that this physical character of these stones is related to the phytoestrogen substances of the clover.

Prolonged obstruction of the pylorus causes sequestration of the fluid into the forestomach with subsequent dehydration, absence of urination and defecation, and abdominal distention. These signs similar to those of urine retention (Radostits, et al., 2000). However, catheterization and flushing of the bladder could differentiate between both affections.

With respect to cystitis, it is a common affection of female than male as a result of the short urethra and its close location to the uterus, that predispose female more than male to cystitis(Kelly, 1984; and Radostits, et al., 2000), however, despite of that, male still a subject of cystitis too. Cystitis can be treated by massive doses of antibiotics. However, sensitivity test is a very helpful procedure for determination of the antibiotic of choice. Collection of urine sample, under aseptic condition (Radostits, et al., 2007), from the bladder was very helpful in determining suitable antibiotic, shortening the period and course of treatment, rapid improvement of cases, and determining suitable time for discontinuation of treatment.

It is concluded that retrograde catheterization can be performed in cattle and buffalo calves before and after separation of glans penis and it is a very helpful technique to diagnose and treat many affections under field condition. The technique is safe, applicable, simple, cheap, diagnostic and save the use of complicated or expensive equipments for diagnosis of urethral and bladder affections in cattle and buffalo calves before and after separation of glans penis.

\section{REFERENCES}

Abd Elfattah, M. and Sedeek, A. (2005): Dorsal versus ventral urethrotomy technique for treatment of obstructive urethrolithiasis in cattle calves. Assiut Veterinary Medical Journal 51, 198-209.

Bezuidenhout, A. and Coetzer, D. (1982): The urethral diverticulum of the bull. Journal of South Africa Veterinary Association 53, 275-276.

Blood, D. and Henderson, J. (1976): Veterinary medicine. $4^{\text {th }}$ Ed. ELBS, Bailliere Tendall, London.

Bryden, D. (1984): University Sydney, Post Grad., Cttee Vet. Sci., Vade Mecum, 4: 73-74. 
Garrett, P. (1987): Urethral recess in male goats, sheep, cattle, and swine. Journal of the American Veterinary Medical Association 191, 689-691.

Gasthuys, F.; Steenhaut, M.; De Moor, A. and Sercu, K. (1993): Surgical treatment of urethral obstruction due to urolithiasis in male cattle: a review of 85 cases. Vet. Record. 133: 522-526

Hall, L. and Clarke, K. (1983): Veterinary anesthesia. $8^{\text {th }}$ Ed., ELBS and Bailliere Tindall.

Halland, S.; House, J. and George, L. (2002): Urethroscopy and laser lithotripsy for the diagnosis and treatment of obstructive urolithiasis in goats and pot-bellied pigs. Journal of the American Veterinary Medical Association 220, 1831-1834.

Hinkle, R.; Howard, J. and Stowater, J. (1978): An anatomic barrier to urethral catheterization in the male goat. Journal of the American Veterinary Medical Association 173, 1584-1586.

Hungerford, T. (1990): Diseases of livestock, $9^{\text {th }}$ Ed., Lindsay Castelloe, McGrew Hill Book Co., Australia.

Ibrahim, M. (2002): A study on zinc metabolism in goats. MVSc thesis, Nutrition and clinical nutrition, Cairo Univ., Beni-Suef branch, Egypt.

Kelly, W. (1984): Veterinary clinical diagnosis. The urinary system. $3^{\text {rd }}$ Ed. Baillier Tindall, London, UK.

Lavania, J. and Angelo, S. (1975): Observations on experimental perineal exteriorisation of the bovine urinary bladder. Veterinary Record. 97, 261-262.

Nancy, W. (1995): Urolithiasis in Pygmy goat. Pygmy Goat World Magazine. http://kinne.net/urincalc.htm. Accessed 1/11/2008. Accessed 20/8/2007

Radostits, O.; Gay, C.; Hinchcleff, B. and Constable, P. (2007): Veterinary medicine, A textbook of the diseases of cattle, sheep, pigs, goats, and horses. $10^{\text {th }}$ Ed., Saunders Elsevier, London.

Radostits, O.; Mayhew, I. and Huston, D. (2000): Veterinary clinical examination and diagnosis. Harcourt publisher Ltd., W.B. Saunders, London, UK.

Rosenberger, G.; Dirksen, G.; Gruder, A.; Eberhard, G.; Krause, D. and Stober, M. (1979): Clinical examination of cattle. $2^{\text {nd }} E d$. Verlag Paul Parey, Berlin and Hamburg, D-1000 Berline, Germany. 
Seif, M.; Abdel Fattah, M. and Sedeek, A. (2005): Permanent urinary diversion in ruminants. Assiut Veterinary Medical Journal 51, 210-223.

Seif, M.; Sedeek, A.; Abdel-Fattah, M. and Bakr, H. (2007): Obstructive urethrolithiasis in cattle calves: (Areview of 319 cass). $9^{\text {th }}$ Sci. Cong., Egyptian Society For Cattle Diseases, 2-4 Dec., Assiut, Egypt, 306-323.

Shokry, M. and Al-Saadi, H. (1980): Retractor penis myotomy for catheterization in sheep and goats. Modern Veterinary Practice 61, 700-705.

Timm, K. and Watrous, B. (1988): Urethral recess in two male llamas. Journal of the American Veterinary Medical Association 192, 937-938.

Van Metre, D. (1987): Personal communication with Sedeek A. M.

Van Metre, D. and Smith, B. (1991): Clinical management of urolithiasis in small ruminants. Transactions of the CA and NV VMA Scientific Seminar, Reno, NV, 204-215.

Van Weeren, P.; Klein, W. and Voorhout, G. (1987): Urolithiasis in small ruminants. II. Cysto-urethrography as a new aid in diagnosis. Veterinary Quaternary 9, 79-83. 